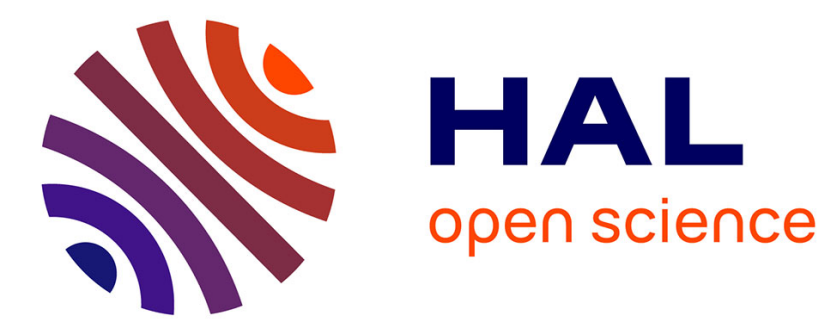

\title{
Trace theorem on the Heisenberg group
}

Hajer Bahouri, Jean-Yves Chemin, Chao-Jiang Xu

\section{To cite this version:}

Hajer Bahouri, Jean-Yves Chemin, Chao-Jiang Xu. Trace theorem on the Heisenberg group. Annales de l'Institut Fourier, 2009, 59 (2), pp.491-514. hal-00434256

\section{HAL Id: hal-00434256 https://hal.science/hal-00434256}

Submitted on 23 Nov 2009

HAL is a multi-disciplinary open access archive for the deposit and dissemination of scientific research documents, whether they are published or not. The documents may come from teaching and research institutions in France or abroad, or from public or private research centers.
L'archive ouverte pluridisciplinaire HAL, est destinée au dépôt et à la diffusion de documents scientifiques de niveau recherche, publiés ou non, émanant des établissements d'enseignement et de recherche français ou étrangers, des laboratoires publics ou privés. 


\title{
TRACE THEOREM ON THE HEISENBERG GROUP
}

\author{
HAJER BAHOURI, JEAN-YVES CHEMIN, AND CHAO-JIANG XU
}

\begin{abstract}
We prove in this work the trace and trace lifting theorem for Sobolev spaces on the Heisenberg groups for hypersurfaces with characteristics submanifolds.
\end{abstract}

Résumé : Dans ce travail, nous démontrons des théorèmes de trace et de relèvement pour les espaces de Sobolev sur le groupe de Heisenberg pour des hypersurfaces dont l'ensemble caractéristique est une sous-variété.

Key words Trace and trace lifting, Heisenberg group, Hörmander condition, Hardy's inequality

A.M.S. Classification $35 \mathrm{~A}, 35 \mathrm{H}, 35 \mathrm{~S}$.

\section{INTRODUCTION}

In this work, we proceed with the study of the problem of restriction of functions that belongs to Sobolev spaces associated to left invariant vector fields for the Heisenberg group $\mathbb{H}^{d}$. We shall assume that $d \geq 2$. Let us recall that the Heisenberg group is the space $\mathbb{R}^{2 d+1}$ of the (non commutative) law of product

$$
w \cdot w^{\prime}=(x, y, s) \cdot\left(s^{\prime}, x^{\prime}, y^{\prime}\right)=\left(x+x^{\prime}, y+y^{\prime}, s+s^{\prime}+\left(y \mid x^{\prime}\right)-\left(y^{\prime} \mid x\right) .\right.
$$

The left invariant vector fields are

$$
X_{j}=\partial_{x_{j}}+y_{j} \partial_{s}, \quad Y_{j}=\partial_{y_{j}}-x_{j} \partial_{s}, j=1, \cdots, d \quad \text { and } \quad S=\partial_{s}=\frac{1}{2}\left[Y_{j}, X_{j}\right] .
$$

In all that follows, we shall denote by $\mathcal{Z}$ this family and state $Z_{j}=X_{j}$ and $Z_{j+d}=Y_{j}$ for $j$ in $\{1, \cdots, d\}$. Moreover, for any $C^{1}$ function $f$, we shall state

$$
\nabla_{\mathbb{H}} f \stackrel{\text { def }}{=}\left(Z_{1} \cdot f, \cdots, Z_{2 d} \cdot f\right) .
$$

The key point is that $\mathcal{Z}$ satisfies Hörmander's condition at order 2, which means that the family $\left(Z_{1}, \cdots, Z_{2 d},\left[Z_{1}, Z_{d+1}\right]\right)$ spans the whole tangent space $T \mathbb{R}^{2 d+1}$.

For $k \in \mathbb{N}$ and $V$ an open subset of $\mathbb{H}^{d}$, we define the associated Sobolev space as following

$$
H^{k}\left(\mathbb{H}^{d}, V\right)=\left\{f \in L^{2}\left(\mathbb{R}^{2 d+1}\right) / \operatorname{Supp} f \subset V \quad \text { and } \quad \forall \alpha /|\alpha| \leq k, Z^{\alpha} f \in L^{2}\left(\mathbb{R}^{2 d+1}\right)\right\},
$$

where if $\alpha \in\{1, \cdots, 2 d\}^{k^{\prime}},|\alpha| \stackrel{\text { def }}{=} k^{\prime}$ and $Z^{\alpha} \stackrel{\text { def }}{=} Z_{\alpha_{1}} \cdots Z_{\alpha_{k^{\prime}}}$. As in the classical case, when $s$ is any real number, we can define the function space $H^{s}\left(\mathbb{H}^{d}\right)$ through duality and complex interpolation, Littlewood-Paley theory on the Heisenberg group (see [4]), or Weyl-Hörmander calculus (see [8], [10] and [11]).

It turns out that these spaces have properties which look very much like the ones of usual Sobolev spaces, see [4] and their references.

The purpose of this paper is the study of the problems of trace and trace lifting on a smooth hypersurface of $\mathbb{H}^{d}$ in the frame of Sobolev spaces. Let us point out that the problem of existence of trace appears only when $s$ is less than or equal to 1 . Indeed, under the subellipicity of system $\mathcal{Z}$, the space $H^{s}\left(\mathbb{H}^{d}\right)$ is included locally in $H^{\frac{s}{2}}\left(\mathbb{R}^{2 d+1}\right)$. So if $s$ is strictly 
larger than 1, this implies that the trace on any smooth hypersurface exists and belongslocally to the usual Sobolev space $H^{\frac{s}{2}-\frac{1}{2}}$ of the hypersurface. Thus the case when $s=1$ appears as the critical one. It is the case we study here.

1.1. Statement of the results. Two very different cases then appear: the one when the hypersurface is non characteristic, which means that any point $w_{0}$ of the hypersurface $\Sigma$ is such that $\mathcal{Z}_{\mid w_{0}} \not \subset T_{w_{0}} \Sigma$, and the one when some point $w_{0}$ of the hypersurface $\Sigma$ is characteristic, which means that $\mathcal{Z}_{\mid w_{0}} \subset T_{w_{0}} \Sigma$.

The non characteristic case is now well understood. In [4], we give a full account of trace and trace lifting results on smooth non characteristic hypersurfaces for $s \geq 1 / 2$. This result generalize various previous results (see among others [7], [12] and [21]).

Let us recall this theorem in the case of $H^{1}$ (see [4] for the details). If $w_{0}$ is any non characteristic point of $\Sigma$, then there exists at last one of the vector fields $Z_{1}, \cdots Z_{2 d}$ which is transverse to $\Sigma$ at $w_{0}$. We denote by $\mathcal{X}_{\Sigma}$ the subspace of $T \Sigma$ define, for $w$ in $\Sigma$, by $\mathcal{X}_{\left.\Sigma\right|_{w}}=$ $\left.T_{w} \Sigma \cap \mathcal{X}\right|_{w}$ where $\mathcal{X}$ is the $C^{\infty}$-module of vector fields spanned by $\left\{Z_{1}, \cdots, Z_{2 d}\right\}$. It is easily checked that, if $g$ is a local defining function of $\Sigma$, the family

$$
R_{j, k} \stackrel{\text { def }}{=}\left(Z_{j} \cdot g\right) Z_{k}-\left(Z_{k} \cdot g\right) Z_{j}
$$

generates $\mathcal{X}_{\Sigma}$ and that it satisfies the Hörmander condition at order 2 (see for instance Lemma 4.1 of [4]). We define

$$
H^{k}\left(\Sigma, Z_{\Sigma}\right)=\left\{f \in L^{2}(\Sigma) / \operatorname{Supp} f \subset V \text { and } \forall(j, k), R_{j, k} u \in L^{2}\right\} .
$$

We have proved the following trace and trace lifting theorem in [4]:

Theorem 1.1. Let us suppose that $\Sigma$ is non characteristic on an open subset $V$ of $\mathbb{H}^{d}$, then the trace operator on $\Sigma$ denoted by $\gamma_{\Sigma}$ is an onto continuous map from $H^{1}\left(\mathbb{H}^{d}, V\right)$ onto $\left[H^{1}\left(\Sigma, Z_{\Sigma}\right), L^{2}(\Sigma)\right]_{\frac{1}{2}} \stackrel{\text { def }}{=} H^{\frac{1}{2}}\left(\Sigma, Z_{\Sigma}\right)$.

Remark As the system $\mathcal{Z}_{\Sigma}$ satisfies the Hörmander's condition at order 2, Theorem 1.1 implies in particular that $\gamma_{\Sigma}$ maps $H^{1}\left(\mathbb{H}^{d}, V\right)$ into $H^{1 / 4}(\Sigma, V)$.

We shall now consider the characteristic case. The set of characteristic points of $\Sigma$

$$
\Sigma_{c}=\left\{w \in \Sigma / \mathcal{Z}_{\mid w} \subset T_{w} \Sigma\right\},
$$

may have a complicated structure. Let us introduce the following definition.

Definition 1.1. A characteristic point $w_{0}$ of a hypersurface $\Sigma$ is a regular point of order $r$ if and only if

i) for any 1-form $\theta \in T^{\star} \mathbb{R}^{2 d+1}$ that vanishes on $T \Sigma$ and such that $\theta\left(w_{0}\right) \neq 0$, the system $\left(\mathcal{L}_{Z_{j}} \theta_{\mid T_{w_{0}} \Sigma}\right)_{1 \leq j \leq 2 d}$ is of rank $r$;

ii) near $w_{0}$, the characteristic set $\Sigma_{c}$ is a submanifold of $\Sigma$ of codimension $r$ in $\Sigma$.

Let us make some comments about this definition. A regular characteristic point of order $2 d$ is exactly the familiar notion of non degenerate characteristic point. This notion of non degenerate characteristic point have been used in our preceeding work [4] to study this problem of trace.

As wee shall prove in forthcoming Proposition 2.1, if $g$ is a local defining function of $\Sigma$, the condition ii) means exactly that the matrix $\left(Z_{i} \cdot Z_{j} \cdot g\right)_{1 \leq i, j \leq 2 d}$ is of rank $r$ at $w_{0}$. Let us notice that, because, if $i \in\{1, \cdots d\}$ and $j \neq i+d$,

$$
\left(Z_{i} \cdot Z_{i+d} \cdot g\right)\left(w_{0}\right)-\left(Z_{i+d} \cdot Z_{i} \cdot g\right)\left(w_{0}\right)=-2 \partial_{s} g\left(w_{0}\right) \neq 0 \quad \text { and } \quad\left(Z_{i} \cdot Z_{j} \cdot g\right)=\left(Z_{j} \cdot Z_{i} \cdot g\right) \text {, }
$$

the rank of the matrix $\left(Z_{i} \cdot Z_{j} \cdot g\right)_{1 \leq i, j \leq 2 d}$ is at least $d$ at $w_{0}$. 
Let us give some examples. First let us consider the case when the hypersurface $\Sigma$ is give by an equation of the type $s-P(x, y)$ where $P$ is a homogenenous polynomial of degree 2 on $\mathbb{R}^{2 d}$. Let us observe that this equation is homogenenous of order 2 wih respect to the dilation of Heisenberg group $d_{\lambda}(x, y, s) \stackrel{\text { def }}{=}\left(\lambda x, \lambda y, \lambda^{2} s\right)$. In this case $w_{0}=(0,0,0)$ is always a regular characteristic point. Indeed the family $\left(Z_{j} \cdot g\right)_{1 \leq j \leq 2 d}$ is a family of linear form on $\mathbb{R}^{2 d}$. As $X_{j \mid w_{0}}=\partial_{x_{j}}$ and $Y_{j w_{0}}=\partial_{y_{j}}$, the rank of the family is exactly the rank of the matrix $\left(Z_{i} \cdot Z_{j} \cdot g\right)_{1 \leq i, j \leq 2 d}$ at point $w_{0}$. Thus $\Sigma_{c}$ is obviously a submanifold of codimension $r$ of $\Sigma$.

Now let us exhibit an example of non regular characteristic point. In the case when $d=2$, let us define, for $\lambda$ in $\mathbb{R}$,

$$
\Sigma_{\lambda}=\left\{\left(x_{1}, y_{1}, x_{2}, y_{2}, s\right) \in \mathbb{R}^{5} / s=x_{1} y_{1}+\lambda\left(x_{1}^{3}+y_{1}^{3}\right)\right\} .
$$

If $\lambda=0$, as observe above, the origin is a regular characteristic point. A very easy computation shows that the rank of the matrix $\left(Z_{i} \cdot Z_{j} \cdot g\right)_{1 \leq i, j \leq 4}$ is three. But the characteristic set $\Sigma_{\lambda, c}$ is the set of points of $\Sigma_{\lambda}$ such that

$$
3 \lambda x_{1}^{2}=-2 x_{1}+3 \lambda y_{1}^{2}=y_{2}=x_{2}=0 .
$$

If $\lambda \neq 0$, the characteristic set $\Sigma_{\lambda, c}$ reduces to the origin.

Let us introduce some rings of functions adapted to our situation.

Definition 1.2. Let $W$ be any open subset of $\Sigma$ and $F$ a closed subset of $W$. Let us denote by $C_{F}^{\infty}(W)$ the set of smooth functions $a$ on $W \backslash F$ such that for any multi-index $\alpha$, a constant $C_{\alpha}$ exists such that

$$
\forall \alpha \in \mathbb{N}^{d}\left|\partial^{\alpha} a(z)\right| \leq C_{\alpha} d(z, F)^{-|\alpha|},
$$

where $d$ denotes the distance on $\Sigma$ induced by the euclian distance on $\mathbb{R}^{2 d+1}$.

Now let us define the vector fields on $\Sigma$ which will describe the regularity on $\Sigma$.

Definition 1.3. Let $w_{0}$ a characteristic point of a hypersurface $\Sigma$. Let $W$ be a neighhourhood of $w_{0}$. We denote by $Z_{\Sigma}$ the $C_{\Sigma_{c}}^{\infty}(W)$ modulus spanned by the set vector fields of $\mathcal{Z} \cap T \Sigma_{\mid W}$ that vanish on $\Sigma_{c}$.

As we shall see in Proposition 3.1, the modulus $Z_{\Sigma}$ is a finite type (of course as a $C_{\Sigma_{c}}^{\infty}(W)$ modulus) if $w_{0}$ is a regular characteristic point and $W$ is choosen small enough. If $g$ is a local defining function of $\Sigma$, a generating system is given by

$$
R_{j, k} \stackrel{\text { def }}{=}\left(Z_{j} \cdot g\right) Z_{k}-\left(Z_{k} \cdot g\right) Z_{j} \quad \text { for } \quad 1 \leq j \leq k \leq 2 d .
$$

Now we are ready to introduce the space of traces.

Definition 1.4. Let $w_{0}$ a regular characteristic point of a hypersurface $\Sigma$. Let $W$ be a small enough neighbourhood of $w_{0}$. We denote by $H^{1}\left(\mathcal{Z}_{\Sigma}, W\right)$ the space of functions $v$ of $L^{2}(\Sigma)$ supported in $W$ such that

$$
\|v\|_{H^{1}\left(\mathcal{Z}_{\Sigma}\right)}^{2} \stackrel{\text { def }}{=}\|v\|_{L^{2}(\Sigma)}^{2}+\sum_{1 \leq j, k \leq 2 d}\left\|R_{j, k} v\right\|_{L^{2}(\Sigma)}^{2} \leq \infty
$$

where the family $\left(R_{j, k}\right)_{1 \leq j, k \leq 2 d}$ is given by (1.1). If $s \in[0,1]$, we define $H^{s}\left(\mathcal{Z}_{\Sigma}, V\right)$ by complex interpolation.

Our theorem is the following. 
Theorem 1.2. Let $w_{0}$ a regular characteristic point of a hypersurface $\Sigma$. Let $V$ be a small enough neighhourhood of $w_{0}$. Then the restriction map $\gamma_{\Sigma}$ is an onto continuous map from $H^{1}\left(\mathbb{H}^{d}, V\right)$ onto $H^{\frac{1}{2}}\left(\mathcal{Z}_{\Sigma}, V \cap \Sigma\right)$.

Let us remark that, if $w_{0}$ is a non degenerate characteristic point (i.e. a regular characterisitic point or order $2 d$ ) this theorem is Theorem 1.8 of [4].

1.2. Structure of the proof. In our paper [4], we use a blow up of the point $w_{0}$ (which is $\Sigma_{c}$ in the case when the characteristic point $w_{0}$ is of order $2 d$ ). Here we shall blow up the submanifold $\Sigma_{c}$. In order to do it, let us introduce a function $\varphi \in \mathcal{D}\left(\mathbb{R}_{+} \backslash\{0\}\right)$ such that

$$
\forall t \in[-1,1] \backslash\{0\}, \sum_{p=0}^{\infty} \varphi\left(2^{p} t\right)=1 .
$$

Let us define the function $\rho_{c}$ by $\rho_{c} \stackrel{\text { def }}{=}\left(g^{2}+\left|\nabla_{\mathbb{H}} g\right|^{4}\right)^{\frac{1}{4}}$. Now writing that for any function $u$ in $L^{2}\left(\rho_{c} \leq 1\right)$,

$$
u=\sum_{p=0}^{\infty} \varphi_{p} u \quad \text { with } \quad \varphi_{p}(w) \stackrel{\text { def }}{=} \varphi\left(2^{p} \rho_{c}(w)\right),
$$

we apply Theorem 1.1 of trace and trace lifting to each piece $\varphi_{p} u$ which is supported in a domain where $\Sigma$ is non charactersitic because $\rho_{c} \sim 2^{-p}$ in this domain. This decomposition leads immediately to the problem of estimating the norm $H^{1}\left(\mathbb{H}^{d}\right)$ of each piece $\varphi_{p} u$. Leibnitz formula and the chain rule tell us that

$$
\nabla_{\mathbb{H}}\left(\varphi_{p} u\right)=\varphi_{p} \nabla_{\mathbb{H}} u+2^{p} \varphi^{\prime}\left(2^{p} \rho_{c}\right) u \nabla_{\mathbb{H}} \rho_{c} .
$$

Let us observe that, as

$$
Z_{j} \rho_{c}^{4}=2 g Z_{j} \cdot g+4\left|\nabla_{\mathbb{H}} g\right|^{2}\left(Z_{j} \cdot g\right) \sum_{k=1}^{2 d} Z_{j} \cdot\left(Z_{k} \cdot g\right),
$$

we have, for any real number $s,\left|\nabla_{\mathbb{H}} \rho_{c}^{s}\right| \leq C_{s} \rho_{c}^{s-1}$. As the support of $\varphi^{\prime}\left(2^{p} \rho_{c}\right)$ included in $\rho_{c} \sim 2^{-p}$, the supports of $\varphi^{\prime}\left(2^{p} \rho_{c}\right)$ and $\varphi^{\prime}\left(2^{p^{\prime}} \rho_{c}\right)$ are disjoint if $\left|p-p^{\prime}\right| \leq N_{0}$ for some $N_{0}$. Thus, we get that

$$
\sum_{p=0}^{\infty} 2^{2 p}\left\|\varphi^{\prime}\left(2^{p} \rho_{c}\right) u \nabla_{\mathbb{H}} \rho_{c}\right\|_{L^{2}}^{2} \leq C\left\|\frac{u}{\rho_{c}}\right\|_{L^{2}}^{2} .
$$

This leads to the proof of the following Hardy type inequality.

Theorem 1.3. If $w_{0}$ is a regular characteristic point of $\Sigma$, a neighbourhood $V$ of $w_{0}$ exists such that, for any $u$ in the space $H^{1}\left(\mathbb{H}^{d}, V\right)$ of $H^{1}\left(\mathbb{H}^{d}\right)$ functions supported in $V$,

$$
\int_{\mathbb{H}^{d}} \frac{u^{2}}{\rho_{c}^{2}} d w \leq C\left\|\nabla_{\mathbb{H}} u\right\|_{L^{2}}^{2} . \quad \text { with } \quad \rho_{c}=\left(g^{2}+\left|\nabla_{\mathbb{H}} g\right|^{4}\right)^{\frac{1}{4}} .
$$

This theorem implies that, for any $u$ in $H^{1}\left(\mathbb{H}^{d}, V\right)$,

$$
\sum_{p=0}^{\infty}\left\|\nabla_{\mathbb{H}}\left(\varphi_{p} u\right)\right\|_{L^{2}}^{2} \leq C\left\|\nabla_{\mathbb{H}} u\right\|_{L^{2}}^{2} .
$$

The proof of this theorem, which is the core of this work, is the purpose of the second section.

In the third section, we first straighten the submanifolds $\Sigma$ and $\Sigma_{c}$, and after dilation, we apply Theorem 1.1. This gives a rather unpleasant description on the trace space. Then, we prove an interpolation result which allows to conclude the proof of Theorem 1.2. 


\section{A Hardy type inequality}

2.1. The classical Hardy inequality. As a warm up, let us recall briefly the usual proof of the classical Hardy inequality ${ }^{1}$.

$$
\int_{\mathbb{H}^{d}} \frac{u^{2}}{\rho^{2}} d w \leq C\left\|\nabla_{\mathbb{H}} u\right\|_{L^{2}}^{2} \quad \text { with } \quad \rho(w)=\left(s^{2}+\left(|x|^{2}+|y|^{2}\right)^{2}\right)^{\frac{1}{4}} .
$$

As $\mathcal{D}\left(\mathbb{H}^{d} \backslash\{0\}\right)$ is dense $H^{1}\left(\mathbb{H}^{d}\right)$, we have restrict ourselves to functions $u$ in $\mathcal{D}\left(\mathbb{H}^{d} \backslash\{0\}\right)$. Then the proof mainely consists in an integration by parts with respect to the radial vector field $R_{\mathbb{H}}$ adapted to the structure of $\mathbb{H}^{d}$, namely

$$
R_{\mathbb{H}} \stackrel{\text { def }}{=} 2 s \partial_{s}+\sum_{j=1}^{d}\left(x_{j} \partial_{x_{j}}+y_{j} \partial_{y_{j}}\right)=s\left[Y_{1}, X_{1}\right]+\sum_{j=1}^{d}\left(x_{j} X_{j}+y_{j} Y_{j}\right)
$$

once noticed that $R_{\mathbb{H}} \cdot \rho^{-2}=-2 \rho^{-2}$ and $\operatorname{div} R_{\mathbb{H}}=2 d+2$. More precisely, this gives

$$
-d \int \frac{u^{2}}{\rho^{2}} d w=\int \sum_{j=1}^{d} \frac{u}{\rho}\left(\frac{x_{j}}{\rho} X_{j}+\frac{y_{j}}{\rho} Y_{j}\right) u d w-\int\left(Y_{1} \frac{s}{\rho^{2}}\right) u\left(X_{1} u\right) d w+\int\left(X_{1} \frac{s}{\rho^{2}}\right) u\left(Y_{1} u\right) d w .
$$

As we have $\left|Z_{j}\left(\frac{s}{\rho^{2}}\right)\right| \leq C \rho^{-1}$, Cauchy-Schwarz inequality gives (2.5).

2.2. Construction of substitute of $\rho$ and $R_{\mathbb{H}}$. Let us start with some remarks about the relations between $\Sigma_{c}$ and the vector fileds $Z_{j}$ in the case when $w_{0}$ is a regular characteristic point.

Proposition 2.1. The condition ii) of Definition 1.1 is equivalent to the fact that, for any defining function $g$ of $\Sigma$, the rank of the matrix $\left(Z_{i} \cdot Z_{j} \cdot g\right)_{1 \leq i, j \leq 2 d}$ is $r$.

Proof of Proposition 2.1 Let $g$ be a local defining function of $\Sigma$. Of course, $D g$ vanishes on $T \Sigma$. As $Z_{j}\left(w_{0}\right)$ belongs to $T_{w_{0}} \Sigma$, we have $\mathcal{L}_{Z_{j}}(D g)\left(w_{0}\right)=D\left(Z_{j} \cdot g\right)\left(w_{0}\right)$. By definition of $\mathcal{Z}$, we infer that

$$
D\left(Z_{j} \cdot g\right)\left(w_{0}\right)=\sum_{i=1}^{2 d}\left(Z_{i} \cdot Z_{j} \cdot g\right)\left(w_{0}\right) d z_{i}
$$

Thus the rank of matrix $\left(Z_{i} \cdot Z_{j} \cdot g\right)_{1 \leq i, j \leq 2 d}$ is the rank of $\mathcal{L}_{Z_{j}}(D g)\left(w_{0}\right)$.

Conversevely, let $\theta$ be a 1 -form that vanishes on $T \Sigma$ and such that $\theta\left(w_{0}\right) \neq 0$ and $g$ a local defining function of $\Sigma$. A function $a$ that does not vanish at $w_{0}$ exists such that $\theta=a D g$. Thanks to Leibnitz formula, $\mathcal{L}_{Z_{j}}(\theta)\left(w_{0}\right)_{\mid T_{w_{0}} \Sigma}=a\left(w_{0}\right) D\left(Z_{j} \cdot g\right)\left(w_{0}\right)_{\mid T_{w_{0}} \Sigma}$. The fact that the function $a$ does not vanish at point $w_{0}$ implies the proposition.

In all that follows, $g$ will denote a defining function of $\Sigma$ of the form $g(x, y, s)=s+f(x, y)$ (this is allowed by the implit function theorem) near the origin $w_{0}$ of $\mathbb{H}^{d}$ which is assumed to be a characterisitc regular point of order $r \leq 2 d$.

As the matrix $\left(Z_{i} \cdot Z_{j} \cdot g\right)_{1 \leq i, j \leq 2 d}$ is of rank $r$ in $w_{0}$, and as $Z_{i \mid w_{0}}=\partial_{z_{i}}$, a family $\left(j_{\ell}\right)_{1 \leq \ell \leq r}$ exists in $\{1, \ldots, 2 d\}^{r}$ such that the linear forms $\left(D\left(Z_{j_{\ell}} \cdot g\right)\right)_{1 \leq \ell \leq r}$ are linearly independant near $w_{0}$. Moreover, the function $Z_{i} g$ are independant of $s$ and $D g\left(w_{0}\right)=(d s, 0,0)$. Thus the family of functions

$$
\left(g,\left(Z_{j_{1}} \cdot g\right), \cdots,\left(Z_{j_{r}} \cdot g\right)\right)
$$

\footnotetext{
${ }^{1}$ For a different approach based on Fourier analysis, see [3]
} 
is a family of $r+1$ independant functions. They vanish on the submanifold $\Sigma_{c}$ which is by hypothesis a submanifold of $\mathbb{H}^{d}$ of codimension $r+1$. This implies that, near $w_{0}$,

$$
\Sigma_{c}=\left\{w / g(w)=\left(Z_{j_{1}} \cdot g\right)(w)=\cdots=\left(Z_{j_{r}} \cdot g\right)(w)=0\right\} .
$$

We shall keep these notations all along this text.

The definition of substitute to $\rho$ and $R_{\mathbb{H}}$ relies on the following two lemmas.

Lemma 2.1. A couple of vector fields $\left(Z_{0}, \bar{Z}_{0}\right)$ exists in $\left(\mathcal{Z} \backslash\left\{Z_{j_{1}}, \cdots, Z_{j_{r}}\right\}\right) \times( \pm \mathcal{Z})$ such that

$$
\left[Z_{0}, \bar{Z}_{0}\right]=2 \partial_{s} \quad \text { and } \quad D\left(\bar{Z}_{0} \cdot g\right)\left(w_{0}\right) \neq 0 .
$$

Proof of Lemma 2.1 Let us consider $Z_{0} \in \mathcal{Z} \backslash\left\{Z_{j_{1}}, \cdots, Z_{j_{r}}\right\}$. and $\underline{Z}_{0}$ in $\pm \mathcal{Z}$ such that $\left[Z_{0}, \underline{Z}_{0}\right]=2 \partial_{s}$. If $\pm \underline{Z}_{0}$ belongs to $\left\{Z_{j_{1}}, \cdots, Z_{j_{r}}\right\}$, then (2.6) implies that $D\left(\underline{Z}_{0} \cdot g\right)\left(w_{0}\right)$ is different from 0 and then $\bar{Z}_{0}=\underline{Z}_{0}$ fits. If $\pm \underline{Z}_{0}$ is not in $\left\{Z_{j_{1}}, \cdots, Z_{j_{r}}\right\}$, as

$$
\left(Z_{0} \cdot\left(\underline{Z}_{0} \cdot g\right)\right)\left(w_{0}\right)-\left(\underline{Z}_{0} \cdot\left(Z_{0} \cdot g\right)\right)\left(w_{0}\right)=2,
$$

either $D\left(Z_{0} \cdot g\right)\left(w_{0}\right)$ or $D\left(\underline{Z}_{0} \cdot g\right)\left(w_{0}\right)$ is different from 0 . Thus if $D\left(\underline{Z}_{0} \cdot g\right)\left(w_{0}\right)=0$, we get the lemma interchanging the role of $\underline{Z}_{0}$ and $Z_{0}$.

Using (2.6) and (2.7), the proof of the following lemma is very easy and thus omitted.

Lemma 2.2. A neighhourhood $V$ of $w_{0}$ and a family $\left(\alpha_{\ell}\right)_{1 \leq \ell \leq r}$ of functions of $C^{\infty}(V)$ exist such that

$$
Z_{0} \cdot g=\sum_{\ell=1}^{r} \alpha_{\ell}\left(Z_{j_{\ell}} \cdot g\right) .
$$

Now let us state a Hardy inequality, which is obviously better than the one of Theorem 1.3 and which is surprisingly the one we are able to prove.

Theorem 2.1. A neighbourhood $V$ of $w_{0}$ exists such that, for any $u$ in $H^{1}\left(\mathbb{H}^{d}, V\right)$,

$$
\int \frac{u^{2}}{\rho_{0}^{2}} d w \leq C\left\|\nabla_{\mathbb{H}} u\right\|_{L^{2}}^{2} \quad \text { with } \quad \rho_{0} \stackrel{\text { def }}{=}\left(g^{2}+\left(\bar{Z}_{0} \cdot g\right)^{4}\right)^{\frac{1}{4}} .
$$

Now the problem is to find an analogous of $R_{\mathbb{H}}$ is our situation. We do not manage to do it for $\rho_{c}$. For the function $\rho_{0}$, it is done by the following Lemma.

Lemma 2.3. A neighbourhood $V$ of $w_{0}$, two functions $\beta$ and $\theta$ of $C^{\infty}(V)$ exist such that $\theta$ vanishes on $\Sigma_{c}$ and which satisfy the following properties. Let us define

$$
R_{1}=2 g \partial_{s}+\beta\left(\bar{Z}_{0} \cdot g\right) \widetilde{Z}_{0} \quad \text { with } \quad \widetilde{Z}_{0} \stackrel{\text { def }}{=} Z_{0}-\sum_{\ell=1}^{r} \alpha_{\ell} Z_{j \ell}
$$

where the functions $\left(\alpha_{\ell}\right)_{1 \ell \leq r}$ are the functions which appear in Lemma 2.2. Then,

$$
R_{1} \cdot \rho_{0}^{4}=4 \rho_{0}^{4} \quad \text { and } \quad \operatorname{div} R_{1}=3+\theta .
$$

Proof of Lemma 2.3 The main point of the proof is the computation of the function $\beta$. By definition of the function $\rho_{0}$, we have

$$
R_{1} \cdot \rho_{0}^{4}=2 g\left(R_{1} \cdot g\right)+4\left(\bar{Z}_{0} \cdot g\right)^{3}\left(R_{1} \cdot\left(\bar{Z}_{0} \cdot g\right)\right) .
$$

Lemma 2.2 implies that $\widetilde{Z}_{0}$ is tangent to $\Sigma$. Using that $\partial_{s} g \equiv 1$, this implies that $R_{1} \cdot g=2 g$. Let us compute $R_{1} \cdot\left(\bar{Z}_{0} \cdot g\right)$. As $\partial_{s}\left(\bar{Z}_{0} \cdot g\right)=0$, we have

$$
R_{1} \cdot\left(\bar{Z}_{0} \cdot g\right)=\beta\left(\bar{Z}_{0} \cdot g\right)\left(\widetilde{Z}_{0} \cdot\left(\bar{Z}_{0} \cdot g\right)\right)
$$


Let us notice that $Z_{0}$ does not belong to the family $\left(Z_{j_{\ell}}\right)_{1 \leq \ell \leq r}$. Thus $\bar{Z}_{0}$ commutes with the vector fields $Z_{j_{\ell}}$. By definition of $\widetilde{Z}_{0}$, we infer

$$
\begin{aligned}
{\left[\widetilde{Z}_{0}, \bar{Z}_{0}\right] \quad a m p ; } & =a m p ;\left[Z_{0}, \bar{Z}_{0}\right]+\sum_{\ell=1}^{r}\left[\alpha_{\ell} Z_{\ell}, \bar{Z}_{0}\right] \\
a m p & ;=a m p ; 2 \partial_{s}-\sum_{\ell=1}^{r}\left(\bar{Z}_{0} \cdot \alpha_{\ell}\right) Z_{\ell} .
\end{aligned}
$$

Using that $\widetilde{Z}_{0} \cdot g=0$, we deduce

$$
\begin{aligned}
\widetilde{Z}_{0} \cdot\left(\bar{Z}_{0} \cdot g\right) \quad a m p ; & =a m p ; \bar{Z}_{0} \cdot\left(\widetilde{Z}_{0} \cdot g\right)+2 \partial_{s} g-\sum_{\ell=1}^{r}\left(\bar{Z}_{0} \cdot \alpha_{\ell}\right)\left(Z_{\ell} \cdot g\right) \\
a m p & ;=a m p ; 2+\widetilde{\theta} \quad \text { with } \quad \widetilde{\theta} \stackrel{\text { def }}{=}-\sum_{\ell=1}^{r}\left(\bar{Z}_{0} \cdot \alpha_{\ell}\right)\left(Z_{\ell} \cdot g\right) .
\end{aligned}
$$

It turns out that $R_{1} \cdot \rho_{1}^{4}=4 g^{2}+4\left(\bar{Z}_{0} \cdot g\right)^{4} \beta(2+\widetilde{\theta})$. Choosing $\beta \stackrel{\text { def }}{=}(2+\widetilde{\theta})^{-1}$ gives the first relation of Lemma 2.3. Now, let us compute $\operatorname{div} R_{1}$. We have

$$
\operatorname{div} R_{1}=2 \partial_{s} g+\beta \widetilde{Z}_{0} \cdot\left(\bar{Z}_{0} \cdot g\right)+\left(\bar{Z}_{0} \cdot g\right) \operatorname{div} \widetilde{Z}_{0} .
$$

Using that $\partial_{s} g \equiv 1$ and (2.9), we get

$$
\begin{aligned}
\operatorname{div} R_{1} \quad a m p ; & =a m p ; 2+\beta(2+\widetilde{\theta})+\left(\bar{Z}_{0} \cdot g\right) \operatorname{div} \widetilde{Z}_{0} \\
a m p ; & =a m p ; 3+\left(\bar{Z}_{0} \cdot g\right) \operatorname{div} \widetilde{Z}_{0} .
\end{aligned}
$$

This proves the lemma with $\theta \stackrel{\text { def }}{=}\left(\bar{Z}_{0} \cdot g\right) \operatorname{div} \widetilde{Z}_{0}$.

2.3. Proof of Theorem 2.1. Lemma 2.1 implies that, near $w_{0}$, the set $\rho_{0}^{-1}(0)$ is a submanifold of $\mathbb{H}^{d}$ of codimension 2. The following lemma will allow us to assume that $u$ belongs to $\mathcal{D}\left(V \backslash \rho_{0}^{-1}(0)\right)$.

Lemma 2.4. Let $V$ be a bounded domain of $\mathbb{H}^{d}$ and $\Gamma$ is a submanifold of codimension $\geq 2$. Then $\mathcal{D}(V \backslash \Gamma)$ is dense in the space $H_{0}^{1}\left(\mathbb{H}^{d}, V\right)$ of functions of $H_{0}^{1}\left(\mathbb{H}^{d}\right)$ supported in $V$ equipped with the norm

$$
\left(\|u\|_{L^{2}}^{2}+\left\|\nabla_{\mathbb{H}} u\right\|_{L^{2}}^{2}\right)^{\frac{1}{2}} .
$$

Proof of Lemma 2.4 As $H_{0}^{1}\left(\mathbb{H}^{d}, V\right)$ is a Hilbert space, it is enough to prove that the orthogonal of $\mathcal{D}(V \backslash \Gamma)$ is $\{0\}$. Let $u$ be in this space. For any $v$ in $\mathcal{D}(V \backslash \Gamma)$, we have

$$
(u \mid v)_{L^{2}}+\left(\nabla_{\mathbb{H}} u \mid \nabla_{\mathbb{H}} v\right)_{L^{2}}=0 .
$$

By integration by part, this implies that

$$
\forall v \in \mathcal{D}(V \backslash \Gamma),\left\langle u-\Delta_{\mathbb{H}} u, v\right\rangle=0 .
$$

Thus the support of $u-\Delta_{\mathbb{H}} u$ is included in $\Gamma$. As $Z_{j} u$ belongs to $L^{2}$, then $Z_{j}^{2} u$ belongs to $H^{-1}\left(\mathbb{R}^{2 d+1}\right)$ (the classical Sobolev space). And except 0 , no distribution of $H^{-1}\left(\mathbb{R}^{2 d+1}\right)$ can be supported in a submanifold of codimension greater than 1 . Thus $u-\Delta_{\mathbb{H}} u=0$. Taking the $L^{2}$ scalar product with $u$ implies that $u \equiv 0$.

Thanks to Lemma 2.3, we have

$$
\rho_{0}^{-2}=-\frac{1}{2} R_{1} \cdot \rho_{0}^{-2} .
$$


Thus by integration by part, we have, using Lemma 2.3,

$$
\int \frac{u^{2}}{\rho_{0}^{2}} d w=\frac{3}{2} \int \frac{u^{2}}{\rho_{0}^{2}} d w+\int \theta \frac{u^{2}}{\rho_{0}^{2}} d w+I \quad \text { with } \quad I \stackrel{\text { def }}{=} \int \frac{u}{\rho_{0}^{2}}\left(R_{1} \cdot u\right) d w .
$$

Assuming $V$ small enough such that $\|\theta\|_{L^{\infty}(V)} \leq 1 / 4$, we get

$$
\int \frac{u^{2}}{\rho_{0}^{2}} d w \leq 4|I|
$$

In order to estimate $I$, which contains terms of the type $g \partial_{s} u$, we have to compute the vector field $R_{1}$ in term of elements of $\mathcal{Z}$. Using (2.8), we infer that

$$
R_{1}=2 g\left[\widetilde{Z}_{0}, \bar{Z}_{0}\right]+g \sum_{\ell=1}^{r}\left(\bar{Z}_{0} \cdot \alpha_{\ell}\right) Z_{j_{\ell}}+\beta\left(\bar{Z}_{0} \cdot g\right) Z_{0}-\beta\left(\bar{Z}_{0} \cdot g\right) \sum_{\ell=1}^{r} \alpha_{\ell} Z_{j_{\ell}} .
$$

In other terms, two families $\left(\beta_{k}\right)_{1 \leq k \leq 2 d}$ and $\left(\gamma_{k}\right)_{1 \leq k \leq 2 d}$ exist such that

$$
R_{1}=2 g\left[\widetilde{Z}_{0}, \bar{Z}_{0}\right]+\sum_{k=1}^{2 d}\left(\beta_{k} g+\gamma_{k}\left(\bar{Z}_{0} \cdot g\right)\right) Z_{k}
$$

We deduce that

$$
\begin{aligned}
& I \quad a m p ;=\quad a m p ; J_{1}+J_{2} \text { with } \\
& J_{1} \quad a m p ; \stackrel{\text { def }}{=} \quad a m p ; \sum_{k=1}^{2 d} \int \frac{u}{\rho_{0}} \frac{\beta_{k} g+\gamma_{k}\left(\bar{Z}_{0} \cdot g\right)}{\rho_{0}}\left(Z_{k} \cdot u\right) d w \text { and } \\
& J_{2} \quad a m p ; \stackrel{\text { def }}{=} \quad a m p ; \int \frac{u}{\rho_{0}^{2}} g\left[\widetilde{Z}_{0}, \bar{Z}_{0}\right] \cdot u d w .
\end{aligned}
$$

As $V$ is supposed bounded, we have that the functions

$$
\frac{\beta_{k} g+\gamma_{k}\left(\bar{Z}_{0} \cdot g\right)}{\rho_{0}}
$$

are bounded. Cauchy Schwarz inequality yields

$$
\left|J_{1}\right| \leq C\left\|\frac{u}{\rho_{0}}\right\|_{L^{2}}\left\|\nabla_{\mathbb{H}} u\right\|_{L^{2}} .
$$

The estimate about $J_{2}$ is a little bit more difficult to obtain. Let us write that $J_{2}=K_{1}-K_{2}$ with

$$
K_{1} \stackrel{\text { def }}{=} \int \frac{u}{\rho^{2}} g \widetilde{Z}_{0} \cdot\left(\bar{Z}_{0} \cdot u\right) d w \text { and } K_{2} \stackrel{\text { def }}{=} \int \frac{u}{\rho^{2}} g Z_{0} \cdot\left(\widetilde{Z}_{0} \cdot u\right) d w .
$$

By integration by parts, we have $K_{1}=-K_{11}-K_{12}$ with

$$
\begin{gathered}
K_{11} \stackrel{\text { def }}{=} \int \frac{g}{\rho_{0}^{2}}\left(\widetilde{Z}_{0} \cdot u\right)\left(\bar{Z}_{0} \cdot u\right) d w \text { and } \\
K_{12} \stackrel{\text { def }}{=} \int f \frac{u}{\rho_{0}}\left(\bar{Z}_{0} \cdot u\right) d w \quad \text { with } f \stackrel{\text { def }}{=}\left(\operatorname{div} \widetilde{Z}_{0}\right) \frac{g}{\rho_{0}}+\rho_{0}\left(\widetilde{Z}_{0} \cdot \frac{g}{\rho_{0}^{2}}\right) .
\end{gathered}
$$

By definition of $\rho_{0}$, it is obvious that

$$
\left|K_{11}\right| \leq C\left\|\nabla_{\mathbb{H}} u\right\|_{L^{2}}^{2} .
$$

As we can assume that $V$ is included in $\rho^{-1}([0,1])$, we have that $\rho^{-1} g\left|\operatorname{div} \widetilde{Z}_{0}\right| \leq C$ on $V$. Moreover using that $\widetilde{Z}_{0} \cdot g=0$, we get

$$
\left|\widetilde{Z}_{0} \cdot \frac{g}{\rho_{0}^{2}}\right|=\frac{2 g}{\rho_{0}^{6}}\left|\widetilde{Z}_{0} \cdot\left(\bar{Z}_{0} \cdot g\right)\right|\left|\bar{Z}_{0} \cdot g\right|^{3} \leq C \frac{g}{\rho_{0}^{3}} \leq \frac{C}{\rho_{0}} .
$$


This ensures that $f$ is bounded on $V$ and thus by Cauchy-Schwarz inequality,

$$
\left\|K_{12}\right\| \leq C\left\|\frac{u}{\rho_{0}}\right\|_{L^{2}}\left\|\nabla_{\mathbb{H}} u\right\|_{L^{2}} .
$$

Together with (2.14), this proves that

$$
\left|K_{1}\right| \leq C\left(\left\|\frac{u}{\rho_{0}}\right\|_{L^{2}}+\left\|\nabla_{\mathbb{H}} u\right\|_{L^{2}}\right)\left\|\nabla_{\mathbb{H}} u\right\|_{L^{2}} .
$$

In order to estimate $K_{2}$, let us write that, by integration by parts,

$$
K_{2}=\int \frac{g}{\rho_{0}^{2}}\left(\bar{Z}_{0} \cdot u\right)\left(\widetilde{Z}_{0} \cdot u\right) d w+\int \rho_{0}\left(\bar{Z}_{0} \cdot \frac{g}{\rho_{0}^{2}}\right) \frac{u}{\rho_{0}}\left(\widetilde{Z}_{0} \cdot u\right) d w .
$$

Using that

$$
\bar{Z}_{0} \cdot \rho_{0}^{4}=2 g\left(\bar{Z}_{0} \cdot g\right)+4\left(\bar{Z}_{0} \cdot\left(\bar{Z}_{0} \cdot g\right)\right)\left(\bar{Z}_{0} \cdot g\right)^{3},
$$

we immediatly get that the function $\rho_{0}\left(\bar{Z}_{0} \cdot \frac{g}{\rho_{0}^{2}}\right)$ is bounded on $V$ and we deduce that

$$
\left|K_{2}\right| \leq C\left(\left\|\frac{u}{\rho_{0}}\right\|_{L^{2}}+\left\|\nabla_{\mathbb{H}} u\right\|_{L^{2}}\right)\left\|\nabla_{\mathbb{H}} u\right\|_{L^{2}} .
$$

Together with (2.11), (2.13) and (2.15), we infer that

$$
\left\|\frac{u}{\rho_{0}}\right\|_{L^{2}}^{2} \leq C\left(\left\|\frac{u}{\rho_{0}}\right\|_{L^{2}}+\left\|\nabla_{\mathbb{H}} u\right\|_{L^{2}}\right)\left\|\nabla_{\mathbb{H}} u\right\|_{L^{2}}
$$

which concludes the proof of Theorem 2.1.

\section{The PROOF OF THE TRACE AND TRACE LifTiNG THEOREM}

\subsection{Some preliminary properties.}

Proposition 3.1. A neighbourhood $W$ of $w_{0}$ exists such that the $C_{\Sigma_{c}}(W)$ modulus $\mathcal{Z}_{\Sigma}$ spanned the vector fields of $\mathcal{Z} \cap T \Sigma_{\mid W}$ which vanish on the characterisitic submanifold $\Sigma_{c}$ is of finite type and generated by

$$
R_{j, k} \stackrel{\text { def }}{=}\left(Z_{j} \cdot g\right) Z_{k}-\left(Z_{k} \cdot g\right) Z_{j} .
$$

Proof of Proposition 3.1 It is enough to prove that any element $L$ of $\mathcal{Z} \cap T \Sigma$ which vanish on $\Sigma_{c}$ is a combinaison (with coeffcients in $C_{\Sigma_{c}}^{\infty}(W)$ ) of the $R_{j, k}$. By definition

$$
L=\sum_{j=1}^{2 d} \alpha_{j} Z_{j} \quad \text { with } \quad \alpha_{j \mid \Sigma_{c}}=0 \text { and } \sum_{j=1}^{2 d} \alpha_{j}\left(Z_{j} \cdot g\right)=0 .
$$

Let us introduce a partition of unity $\left(\widetilde{\psi}_{j}\right)_{1 \leq j \leq 2 d}$ of the sphere $\mathbb{S}^{2 d-1}$ such that the support of $\widetilde{\psi}_{j}$ is included in the set of $\zeta$ of $\mathbb{S}^{2 d-1}$ such that $\left|\zeta_{j}\right| \geq(4 d)^{-1}$. Let us state

$$
\psi_{j} \stackrel{\text { def }}{=} \widetilde{\psi}_{j}\left(\frac{\nabla_{\mathbb{H}} g}{\left|\nabla_{\mathbb{H}} g\right|}\right) .
$$

It is an exercice left to the reader to check that $\psi_{j}$ belongs to $C_{\Sigma_{c}}^{\infty}(W)$. On $\Sigma \backslash \Sigma_{c}$, we have, for any $j$ in $\{1, \cdots, 2 d\}$,

$$
\psi_{j}(L \cdot g)=\sum_{k=1}^{2 d} \psi_{j} \alpha_{k}\left(Z_{k} \cdot g\right)=0
$$


By definition of $\psi_{j},\left(Z_{j} \cdot g\right)$ does not vanish on the support of $\psi_{j}$. Thus we have

$$
\alpha_{j} \psi_{j}=-\frac{1}{\left(Z_{j} \cdot g\right)} \sum_{k \neq j} \psi_{j} \alpha_{k}\left(Z_{k} \cdot g\right) .
$$

From this, we deduce that

$$
\begin{aligned}
\psi_{j} L \quad a m p & ;=a m p ; \sum_{k \neq j} \psi_{j} \alpha_{k}\left(Z_{k}-\frac{\left(Z_{k} \cdot g\right)}{\left(Z_{j} \cdot g\right)} Z_{j}\right) \\
a m p & ;=a m p ; \sum_{k \neq j} \frac{\psi_{j} \alpha_{k}}{\left(Z_{j} \cdot g\right)}\left(\left(Z_{j} \cdot g\right) Z_{k}-\left(Z_{k} \cdot g\right) Z_{j}\right) .
\end{aligned}
$$

Now the facts that $\alpha_{k} \in C_{\Sigma_{c}}^{\infty}$ and that $\left(Z_{j} \cdot g\right)$ does not vanish on the support of $\psi_{j}$ ensure that

$$
\alpha_{j, k} \stackrel{\text { def }}{=} \frac{\varphi_{j} \alpha_{k}}{\left(Z_{j} \cdot g\right)} \in C_{\Sigma_{c}}^{\infty}
$$

So we have

$$
L=\sum_{1 \leq j \leq k \leq 2 d} \alpha_{j, k}\left(\left(Z_{j} \cdot g\right) Z_{k}-\left(Z_{k} \cdot g\right) Z_{j}\right)
$$

and the proposition is proved.

The blow up prodecure requires to straighten the submanifolds $\Sigma$ and $\Sigma_{c}$.

Lemma 3.1. A neighbourhood $V$ of $w_{0}$ and a diffeomorphism $\chi$ from $V$ onto $\chi(V)$ exist which satisfy the following properties.

- It straighten the submanifolds $\Sigma$ and $\Sigma_{c}$, namely $\chi(\Sigma \cap V)=(s=0) \cap \chi(V)$ and $\quad \chi\left(\Sigma_{c} \cap V\right)=\left(s=z_{1}=\cdots z_{r}=0\right) \cap \chi(W)$.

- The transported vector fields are of the form

$$
\chi^{\star}\left(\partial_{s}\right)=\partial_{s} \quad \text { and } \quad Z_{j}^{D} \stackrel{\text { def }}{=} \chi^{\star}\left(Z_{j}\right)=\frac{\partial}{\partial e_{j}}+\left(\sum_{\ell=1}^{r} \alpha_{k}^{\ell}(z) z_{k}\right) \partial_{s}+h_{j}\left(z, \partial_{z}\right)
$$

where $\left(e_{j}\right)_{1 \leq j \leq 2 d}$ is a basis of $\mathbb{R}^{2 d}$, the $\left(\alpha_{k}^{\ell}\right)$ are smooth bounded functions on $V$ such that, for $j \in\{1, \cdots, r\}, \alpha_{j}^{\ell} \equiv \delta_{j}^{\ell}$ and $\left(h_{j}\right)_{1 \leq j \leq 2 d}$ is a family of smooth vector fields which vanish at $z=0$.

Proof of Lemma 3.1 It is easily checked that the (local) diffeomorphism defined by

$$
\chi(x, y, s)=\left(\begin{array}{ccc}
g(x, y, s)=s+f(x, y) & \\
z_{k}=\left(Z_{j_{\ell}} \cdot g\right)(x, y) & \text { if } & k \leq r \\
z_{k}=\left\langle L_{k},(x, y)\right\rangle & \text { if } \quad k & g t ; r
\end{array}\right)
$$

where the family of linear form $\left(L_{k}\right)_{r+1 \leq k \leq 2 d}$ is choosen such that

$$
\left(D\left(Z_{j_{\ell}} \cdot g\right)\left(w_{0}\right)\right)_{1 \leq \ell \leq r},\left(L_{k}\right)_{r+1 \leq k \leq 2 d}
$$

is a basis of the dual space of $\mathbb{R}^{2 d}$.

From now on, we shall work only in the straighten situation and to avoid excessive heavyness of notations, we shall still denote $Z_{j}^{D}$ by $Z_{j}$. 
3.2. The blow up procedure. Let us write that, for any function $u$, we can write (at least in $L^{2}$ ) that

$$
u=\sum_{p=0}^{\infty} \varphi_{p} u \quad \text { with } \quad \varphi_{p}(z, s) \stackrel{\text { def }}{=} \varphi\left(2^{p}\left(s^{2}+\left|z^{\prime}\right|^{4}\right)^{\frac{1}{4}}\right) \quad \text { and } \quad z^{\prime} \stackrel{\text { def }}{=}\left(z_{1}, \cdots, z_{r}, 0, \cdots 0\right)
$$

where $\varphi$ is the function introduced in (1.2). We shall proof the following theorem.

Theorem 3.1. The restriction map on the hypersurface $(s=0)$ can be extended in a continuous onto map from $H^{1}\left(\mathcal{Z} ;\left\{\rho_{c} \leq 1\right\}\right)$ onto the space $T^{\frac{1}{2}}$ of function $v \in L^{2}\left(\left|z^{\prime}\right| \leq 1\right)$ such that

$$
\|v\|_{T^{\frac{1}{2}}}^{2} \stackrel{\text { def }}{=} \sum_{p=0}^{\infty}\left\|\varphi_{p}^{\Sigma} v\right\|_{H^{\frac{1}{2}}(\mathcal{R}, p)}^{2} \leq \infty \quad \text { with } \quad H^{s}(\mathcal{R}, p) \stackrel{\text { def }}{=}\left[L^{2}\left(2^{-p} \mathcal{C}_{\Sigma}\right), H^{1}\left(\mathcal{R},\left(2^{-p} \mathcal{C}_{\Sigma}\right)\right]_{s} .\right.
$$

where $\mathcal{C}_{\Sigma} \stackrel{\text { def }}{=}\left\{c \leq\left|z^{\prime}\right| \leq C, \varphi_{p}^{\Sigma}(z) \stackrel{\text { def }}{=} \varphi_{p}(z, 0)=\varphi\left(2^{p}\left|z^{\prime}\right|\right),[A, B]_{\theta}\right.$ denotes the complex interpolation between $A$ and $B$ and $H^{1}(\mathcal{R}, W)$ the space of functions of $H^{1}(\mathcal{R})$ supported in $W$.

Proof of Theorem 3.1 Once noticed that the Hardy inequality given by Theorem 1.3 becomes

$$
\int \frac{u^{2}(z, s)}{\left(s^{2}+\left|z^{\prime}\right|^{4}\right)^{\frac{1}{2}}} d z d s \leq C \sum_{j=1}^{2 d}\left\|Z_{j} u\right\|_{L^{2}}^{2},
$$

we get, by computations very similar to the ones done at the beginning of subsection 1.2 , an analogous of (1.4), namely

$$
\sum_{p=0}^{\infty} \sum_{j=1}^{2 d}\left\|Z_{j}\left(\varphi_{p} u\right)\right\|_{L^{2}}^{2} \leq C \sum_{j=1}^{2 d}\left\|Z_{j} u\right\|_{L^{2}}^{2}
$$

Let us notice that outside $\Sigma_{c}=\left\{(z, s) / s=0, z^{\prime}=0\right\}$, thus in particular on the support of $\varphi_{p}$, the hypersurface $\Sigma$ is non characteristic for $\mathcal{Z}$. Thus locally we can apply Theorem 1.1 to each piece $\varphi_{p} u$. The key point is the control of the constant when $p$ tends to $\infty$. In order to do so, it is convenient to use the quasi-homogenenous dilations $\delta_{p}(z, s) \stackrel{\text { def }}{=}\left(2^{p} z, 2^{2 p} s\right)$. Let us define

$$
u_{p}(z, s) \stackrel{\text { def }}{=} \varphi_{0}(z, s) u\left(2^{p} z, 2^{2 p} s\right) \quad \text { and } \quad Z_{j, p} \stackrel{\text { def }}{=} \frac{\partial}{\partial e_{j}}+\sum_{\ell=1}^{r} \alpha_{j}^{\ell}\left(2^{-p} z\right) z_{\ell} \partial_{s}+h_{j}\left(2^{-p} z, \partial_{z}\right) .
$$

It is obvious that a one to one map $\sigma$ of $\{1, \cdots, 2 d\}$ exists such that

$$
\left[Z_{j, p}, Z_{k, p}\right]=2 \delta_{k, \delta(j)} \partial_{s} .
$$

Moreover, as $\left\|u_{p}\right\|_{L^{2}}^{2}=2^{2 p(d+1)}\left\|\varphi_{p} u\right\|_{L^{2}}$, we have, thanks to Hardy inequality (3.16),

$$
\sum_{p=0}^{\infty} 2^{-2 p d}\left\|u_{p}\right\|_{L^{2}}^{2} \leq C \sum_{j=1}^{2 d}\left\|Z_{j} u\right\|_{L^{2}}^{2} .
$$

Applying (3.17), we infer

$$
\sum_{p=0}^{\infty} 2^{-2 p d}\left(\left\|u_{p}\right\|_{L^{2}}^{2}+\sum_{j=1}^{2 d}\left\|Z_{j, p} u_{p}\right\|_{L^{2}}^{2}\right) \leq C \sum_{j=1}^{2 d}\left\|Z_{j} u\right\|_{L^{2}}^{2} .
$$


On the support of $\varphi_{0}$, the hypersurface $(s=0)$ is non characteristic with respect to the family $\left(Z_{j, p}\right)_{1 \leq j \leq 2 d}$ because, for $j$ between 1 and $r$,

$$
Z_{j, p}=\frac{\partial}{\partial e_{j}}+h_{j}\left(2^{-p} z, \partial_{z}\right)+z_{j} \partial_{s}
$$

Let us notice that the transverse component of $Z_{j, p}$ does not depend on $p$. Thus we can apply Theorem 4.6 of [4] together with a result of interpolation between Sobolev space (see Remarque 4.2 page 89 in [8]) to each $u_{p}$. Using that $\left\|u_{p}\right\|_{H^{1}\left(\mathcal{Z}_{p}\right)}=2^{2 p d}\left\|\varphi_{p} u\right\|_{H^{1}(\mathcal{Z})}$, this gives in particular that a constant $C$ exists (independant of $p$ ) such that

$$
\left\|\gamma\left(u_{p}\right)\right\|_{\left[L^{2}\left(\mathbb{R}^{2 d}\right), H^{1}\left(\widetilde{\mathcal{R}}_{p}, \mathbb{R}^{2 d}\right)\right]_{\frac{1}{2}}} \leq C 2^{2 p d}\left\|\varphi_{p} u\right\|_{H^{1}(\mathcal{Z})}
$$

with $\widetilde{\mathcal{R}}_{p}$ is the union of

$$
\mathcal{R}_{p}=\left(\widetilde{\varphi}_{0}^{\Sigma}\left(Z_{j, p} \cdot s\right) Z_{k, p}-\varphi_{0}^{\Sigma}\left(Z_{k, p} \cdot s\right) Z_{j, p}\right)_{1 \leq j, k \leq 2 d} \quad \text { and } \quad\left(\left(1-\widetilde{\varphi}_{0}^{\Sigma}\right) \partial_{j}\right)_{1 \leq j \leq 2 d}
$$

where $\widetilde{\varphi}_{0}^{\Sigma}$ is a smooth function supported in $\mathcal{C}_{\Sigma}$ such that $\widetilde{\varphi}_{0}^{\Sigma} \equiv 1$ near the support of $\varphi_{0}^{\Sigma}$.

At this point, let us recall the definition of complex interpolation. For details of this theory, we refer in particular to [6] and [18].

Definition 3.1. Let $\left(\mathcal{H}_{j},\|\cdot\|_{j}\right)_{j \in\{0,1\}}$ be two Hilbert spaces such that $\mathcal{H}_{1}$ is densely included in $\mathcal{H}_{0}$. Let $\mathcal{F}\left(\mathcal{H}_{0}, \mathcal{H}_{1}\right)$ be the space of holomorphic functions $f$ from the strip $0 \leq \Re e \zeta \leq 1$ into $\mathcal{H}_{0}$ such that $f(j+i t)$ is continuous and vanishes at infinity in $\mathcal{H}_{j}$. Then, for $\left.\theta \in\right] 0,1[$, the space $\left[\mathcal{H}_{0}, \mathcal{H}_{1}\right]_{\theta}$ is

$$
\left[\mathcal{H}_{0}, \mathcal{H}_{1}\right]_{\theta} \stackrel{\text { def }}{=}\left\{v \in L^{2} / \exists f \in \mathcal{F}\left(\mathcal{H}_{0}, \mathcal{H}_{1}\right) / f(\theta)=v\right\}
$$

equipped with the norm

$$
\|v\|_{\left[\mathcal{H}_{0}, \mathcal{H}_{1}\right]_{\theta}} \stackrel{\text { def }}{=} \inf _{f \in \mathcal{F}\left(\mathcal{H}_{0}, \mathcal{H}_{1}\right)} \max _{j \in\{0,1\}} \sup _{t \in \mathbb{R}}\|f(j+i t)\|_{\mathcal{H}_{j}} .
$$

As the support of $\gamma\left(u_{p}\right)$ is included in the support of $\varphi_{0}^{\Sigma}$, let us consider a smooth function $\varphi_{1}^{\Sigma}$ supported in the set where $\varphi_{0}^{\Sigma}$ has value 1 and such that $\varphi_{1}^{\Sigma}$ has value 1 near the support of $\varphi_{0}^{\Sigma}$. If $f$ is a function in $\mathcal{F}\left(L^{2}\left(\mathbb{R}^{2 d}\right), H^{1}\left(\widetilde{\mathcal{R}}_{p}, \mathbb{R}^{2 d}\right)\right)$ such that $f(1 / 2)=v$, then the function $\zeta \mapsto \varphi_{1} f(\zeta)$ belongs to $\mathcal{F}\left(L^{2}\left(\mathcal{C}_{\Sigma}\right), H^{1}\left(\widetilde{\mathcal{R}}_{p}, \mathcal{C}_{\Sigma}\right)\right)$ and $\varphi_{1} f(1 / 2)=v$. As we obviously have that $H^{1}\left(\widetilde{\mathcal{R}}_{p}, \mathcal{C}_{\Sigma}\right)=H^{1}\left(\mathcal{R}_{p}, \mathcal{C}_{\Sigma}\right)$, Inequality $(3.20)$ becomes

$$
\left\|\gamma\left(u_{p}\right)\right\|_{\left[L^{2}\left(\mathcal{C}_{\Sigma}\right), H^{1}\left(\mathcal{R}_{p}, \mathcal{C}_{\Sigma}\right)\right]_{\frac{1}{2}}} \leq C 2^{2 p d}\left\|\varphi_{p} u\right\|_{H^{1}(\mathcal{Z})}
$$

Moreover, dilations on $\mathbb{R}^{2 d}$ of ratio $2^{-p}$ maps $L^{2}\left(\mathcal{C}_{\Sigma}\right)$ (resp. $H^{1}\left(\mathcal{R}_{p}\right), \mathcal{C}_{\Sigma}$ ) into $L^{2}\left(2^{-p} \mathcal{C}_{\Sigma}\right)$ (resp. $H^{1}\left(\mathcal{R}, 2^{-p} \mathcal{C}_{\Sigma}\right)$ ) with norm equal to $2^{-p d}$. Thus by the functorial property of complex interpolation, Inequality (3.21) becomes

$$
\left\|\gamma\left(\varphi_{p} u\right)\right\|_{H^{\frac{1}{2}(\mathcal{R}, p)}} \leq C\left\|\varphi_{p} u\right\|_{H^{1}(\mathcal{Z})} .
$$

Inequality (3.17) implies that $\gamma$ can be extended to a continuous linear map from $H^{1}(\mathcal{Z})$ into $T^{\frac{1}{2}}$.

In order to prove that $\gamma$ is onto, let us consider $v \in T^{\frac{1}{2}}$. By definition of $T^{\frac{1}{2}}$, and after dilation, we infer that

$$
\left\|\varphi_{0}^{\Sigma} v\left(2^{-p} \cdot\right)\right\|_{\left[L^{2}\left(\mathcal{C}_{\Sigma}\right), H^{1}\left(\mathcal{R}_{p}, \mathcal{C}_{\Sigma}\right)\right]_{\frac{1}{2}}} \leq C 2^{p d} c_{p}\|v\|_{T^{\frac{1}{2}}} \quad \text { with } \quad \sum_{p} c_{p}^{2}=1
$$


As $L^{2}\left(\mathcal{C}^{\Sigma}\right)\left(\right.$ resp. $\left.H^{1}\left(\mathcal{R}_{p}, \mathcal{C}_{\Sigma}\right)\right)$ is a subspace of $L^{2}\left(\mathbb{R}^{2 d}\right)\left(\right.$ resp. $\left.H^{1}\left(\widetilde{\mathcal{R}}_{p} ; \mathbb{R}^{2 d}\right)\right)$, using Theorem 4.6 of [4] together with Remarque 4.2 of [8] and (3.23), we claim the existence of a function $\widetilde{u}_{p}$ in the space $H^{1}\left(\widetilde{Z}_{p}\right)$ such that a constant $C$ (independant of $p$ ) exists which satisfies, for any $p$,

$$
\left\|\widetilde{u}_{p}\right\|_{H^{1}\left(\widetilde{\mathcal{Z}}_{p}\right)} \leq C \leq C 2^{p d} c_{p}\|v\|_{T^{\frac{1}{2}}} \quad \text { with } \quad \sum_{p} c_{p}^{2}=1
$$

where $\widetilde{\mathcal{Z}}_{p}$ is the union of the families

$$
\left(\widetilde{\varphi}_{0} Z_{j, p}\right)_{1 \leq j, k \leq 2 d},\left(\left(1-\widetilde{\varphi}_{0}\right) \partial_{j}\right)_{1 \leq j \leq 2 d} \quad \text { and } \quad\left(1-\widetilde{\varphi}_{0}\right) \partial_{s} .
$$

Let us consider a smooth function $\varphi_{1}$ supported in the domain where $\widetilde{\varphi}$ are has value 1 and such that $\varphi_{1} \equiv 1$ near the support of $\varphi_{0}$. Defining $u_{p} \stackrel{\text { def }}{=} \varphi_{1} \widetilde{u}_{p}$, we have, by definition of $\mathcal{R}_{p}$ and by $(3.24)$

$$
u_{p} \in H^{1}\left(\widetilde{\mathcal{Z}}_{p}, \mathcal{C}_{\Sigma}\right)=H^{1}\left(\mathcal{Z}_{p}, \mathcal{C}_{\Sigma}\right) \quad \text { and } \quad\left\|u_{p}\right\|_{H^{1}\left(\mathcal{R}_{p}\right)} \leq C\left\|\widetilde{u}_{p}\right\|_{H^{1}\left(\widetilde{\mathcal{R}}_{p}, \mathcal{C}_{\Sigma}\right)} .
$$

After dilation, this gives

$$
\sum_{p}\left\|u_{p}\left(2^{p} \cdot\right)\right\|_{H^{1}(\mathcal{Z})}^{2} \leq C\|v\|_{T^{\frac{1}{2}}}^{2} .
$$

As an integer $N_{0}$ exists such that

$$
\left|p-p^{\prime}\right| \geq N_{0} \Longrightarrow u_{p}\left(2^{p} \cdot\right) \perp u_{p^{\prime}}\left(2^{p^{\prime}} \cdot\right) \quad \text { in } \quad H^{1}(\mathcal{Z}),
$$

the series $\left(u_{p}\left(2^{p} \cdot\right)\right)_{p}$ converge in $H^{1}(\mathcal{Z})$ to a function $u$ the trace of which is obviously $v$. This concludes the proof of Theorem 3.1.

3.3. The space of trace as an interpolation space. The description given by Theorem 3.1 is not totally satisfactory. We want to describe this space of trace as an interpolation space to get Theorem 1.2. In order to do so, let us define, for $s \in[0,1]$, the space

$$
T^{s} \stackrel{\text { def }}{=}\left\{v \in L^{2} /\|v\|_{T^{s}}^{2} \stackrel{\text { def }}{=} \sum_{p}\left\|\varphi_{p}^{\Sigma} v\right\|_{H^{s}(\mathcal{R}, p)} \leq \infty\right\} .
$$

Let us start with the proof of the following lemma.

Lemma 3.2. The space $T^{1}$ is equal to $H^{1}(\mathcal{R})$ and the norm are equivalents.

Proof of Lemma 3.2 By definition of the norm on $H^{1}\left(\mathcal{R}_{p}\right)$, we have

$$
\left\|\left(\varphi_{p}^{\Sigma} v\right)\left(2^{-p} \cdot\right)\right\|_{H^{1}\left(\mathcal{R}_{p}\right)}^{2}=\left\|\left(\varphi_{p}^{\Sigma} v\right)\left(2^{-p} \cdot\right)\right\|_{L^{2}}^{2}+\sum_{j, k}\left\|\mathcal{R}_{j, k, p}\left(\left(\varphi_{p}^{\Sigma} v\right)\left(2^{-p} \cdot\right)\right)\right\|_{L^{2}}^{2} .
$$

By definition of $R_{j, k, p}$, we have

$$
2^{-2 p d}\left\|\mathcal{R}_{j, k, p}\left(\left(\varphi_{p}^{\Sigma} v\right)\left(2^{-p} \cdot\right)\right)\right\|_{L^{2}}^{2}=\left\|\mathcal{R}_{j, k}\left(\varphi_{p}^{\Sigma} v\right)\right\|_{L^{2}}^{2} .
$$

By Leibnitz formula and by definition of $\varphi_{p}^{\Sigma}$, we have

$$
\begin{aligned}
R_{j, k}\left(\varphi_{p}^{\Sigma}\right)(z) \quad a m p ; & =a m p ; \varphi_{p}^{\Sigma} R_{j, k} \cdot v(z)+\left(R_{j, k} \cdot \varphi_{p}^{\Sigma}\right) v(z) \\
a m p ; & =a m p ; \varphi_{p}^{\Sigma} R_{j, k} \cdot v+2^{p}\left(R_{j, k} \cdot\left|z^{\prime}\right|\right) \varphi^{\prime}\left(2^{p}\left|z^{\prime}\right|\right) .
\end{aligned}
$$

As the vector fields $R_{j, k}$ vanishes at 0 , we have

$$
\sup _{p, j, k}\left\|R_{j, k} \varphi_{p}^{\Sigma}\right\|_{L^{\infty}} \leq \infty .
$$

This gives that

$$
\left|R_{j, k}\left(\varphi_{p}^{\Sigma} v\right)(z)-\varphi_{p}^{\Sigma} R_{j, k} v(z)\right| \leq C \varphi^{\prime}\left(2^{p}\left|z^{\prime}\right|\right)|v(z)| .
$$


As, for some positive integer $N_{0}$, the support of the two functions $\varphi\left(2^{p}\left|z^{\prime}\right|\right)$ and $\varphi\left(2^{p^{\prime}}\left|z^{\prime}\right|\right)$ are disjoint when $\left|p-p^{\prime}\right| \geq N_{0}$, this gives the lemma.

Now Theorem 1.2 will be an easy consequence of the following abstract interpolation lemma.

Lemma 3.3. Let us consider $\left(\mathcal{H}_{j},\|\cdot\|_{j}\right)_{j \in\{0,1\}}$ two Hilbert spaces such that $\mathcal{H}_{1}$ is densely included in $\mathcal{H}_{0}$ and a family $\left(\mathcal{H}_{j, p}\right)_{(j, p) \in\{0,1\} \times \mathbb{N}}$ such that, for any $p, \mathcal{H}_{j, p}$ is a closed subset of $\mathcal{H}_{j}$.

Let us assume that a family of $\left(\Lambda_{p}\right)_{p \in \mathbb{N}}$ of (unbounded) selfadjoints operators on $\mathcal{H}_{0, p}$ exists such that $\mathcal{H}_{1, p}$ equals to the domain of $\Lambda_{p}$ and

$$
\forall u \in \mathcal{H}_{1, p},\|u\|_{\mathcal{H}_{1}} \sim\left\|\Lambda_{p} u\right\|_{\mathcal{H}_{0}} .
$$

Let us assume in addition that a familyof operators $\left(A_{p}\right)_{p \in \mathbb{N}}$ exists such that, for any $(j, p)$ in $\{0,1\} \times \mathbb{N}$, the operator $A_{p}$ is continuous from $\mathcal{H}_{j}$ into $\mathcal{H}_{j, p}$ and

$$
\forall v \in \mathcal{H}_{j}, \lim _{p \infty}\left\|v-\sum_{p=0}^{N} v_{p}\right\|=0 \quad \text { and } \quad\|v\|_{\mathcal{H}_{j}}^{2} \sim \sum_{p}\left\|A_{p} v\right\|_{\mathcal{H}_{j}}^{2} .
$$

Then,

$$
\left[\mathcal{H}_{0}, \mathcal{H}_{1}\right]_{s}=\left\{v \in \mathcal{H}_{0} /\|v\|_{T^{s}}^{2} \stackrel{\text { def }}{=} \sum_{p=0}^{\infty}\left\|A_{p} v\right\|_{\mathcal{H}_{s, p}}^{2}\right\} \quad \text { with } \quad \mathcal{H}_{s, p} \stackrel{\text { def }}{=}\left[\mathcal{H}_{0, p}, \mathcal{H}_{1, p}\right]_{s} .
$$

Proof of Lemma 3.3 It is enough to prove that the two norms are equivalent on the dense space of $v$ such that

$$
v=\sum_{p=0}^{N} v_{p} \quad \text { with } \quad v_{p} \in \mathcal{H}_{1, p} .
$$

Let us first estimate $\|v\|_{\left[\mathcal{H}_{0}, \mathcal{H}_{1}\right]_{s}}$. By definition of the norm on $\mathcal{H}_{s, p}$, a function $f_{p}$ exists in $\mathcal{F}\left(\mathcal{H}_{0, p}, \mathcal{H}_{1, p}\right)$ such that

$$
f_{p}(s)=A_{p} v \quad \text { and } \quad \max _{j \in\{0,1\}} \sup _{t \in \mathbb{R}}\left\|f_{p}(j+i t)\right\|_{\mathcal{H}_{j}} \leq 2\left\|A_{p} v\right\|_{\mathcal{H}_{s, p}} .
$$

Now let us define

$$
F_{N}(\zeta) \stackrel{\text { def }}{=} e^{\zeta^{2}-s^{2}} \sum_{p=0}^{N} f_{p}(\zeta)
$$

As the sum is finite, this is obvious that $F_{N}$ belongs to $\mathcal{F}\left(\mathcal{H}_{0}, \mathcal{H}_{1}\right)$. Because of $(3.26)$, we have, for $j \in\{0,1\}$,

$$
\begin{aligned}
\left\|F_{N}(j+i t)\right\|_{\mathcal{H}_{j}}^{2} \quad a m p ; & \leq a m p ; C e^{-t^{2}} \sum_{p=0}^{N}\left\|f_{p}(j+i t)\right\|_{\mathcal{H}_{j}}^{2} \\
a m p ; & \leq a m p ; C e^{-t^{2}} \sum_{p=0}^{N}\left\|A_{p} v\right\|_{\mathcal{H}_{s, p}}^{2} \\
a m p & \leq a m p ; C\|v\|_{T^{s}}^{2} .
\end{aligned}
$$

Thus by definition of the complex interpolation norm, we deduce that

$$
\|v\|_{\left[\mathcal{H}_{0}, \mathcal{H}_{1}\right]_{s}} \leq C\|v\|_{T^{s}} .
$$


Now let us estimate $\|v\|_{T^{s}}$. In order to do so, let us consider $F$ in $\mathcal{F}\left(\mathcal{H}_{0}, \mathcal{H}_{1}\right)$ such that

$$
F(s)=v \quad \text { and } \max _{j \in\{0,1\}} \sup _{t}\left\|F_{N}(j+i t)\right\|_{\mathcal{H}_{j}} \leq 2\|v\|_{\left[\mathcal{H}_{0}, \mathcal{H}_{1}\right]_{s}} .
$$

For $a$ greater than 1 , let us introduce

$$
\mathcal{N}_{a}(\zeta) \stackrel{\text { def }}{=} e^{\zeta^{2}-s^{2}} \sum_{p=0}^{N} \int_{1}^{a} \lambda^{2 \zeta} d \mu_{p}\left(A_{p} F(\zeta), A_{p} F(z)\right)
$$

where $\mu_{p}$ is the spectral measure of $\Lambda_{p}$. Then, by using (3.25) and (3.26),

$$
\begin{aligned}
\left|\mathcal{N}_{a}(j+i t)\right| \quad a m p ; & \leq a m p ; C e^{-t^{2}}\left|\int_{1}^{a} \lambda^{2 i t} \lambda^{2 j} d \mu_{p}\left(A_{p} F(j+i t), A_{p} F(j+i t)\right)\right| \\
a m p ; & \leq a m p ; C e^{-t^{2}} \sum_{p=0}^{N} \int_{1}^{a} \lambda^{2 j} d \mu_{p}\left(A_{p} F(j+i t), A_{p} F(j+i t)\right) \\
a m p & \leq a m p ; C e^{-t^{2}} \sum_{p=0}^{N}\left\|A_{p} F(j+i t)\right\|_{\mathcal{H}_{j}}^{2} \\
a m p & \leq a m p ; C e^{-t^{2}}\left\|F_{j}(j+i t)\right\|_{\mathcal{H}_{j}}^{2} \\
a m p & \leq a m p ; C\|v\|_{\left[\mathcal{H}_{0}, \mathcal{H}_{1}\right]_{s}}^{2} .
\end{aligned}
$$

Then using the Phragmen-Lindelöf principle, we get that

$$
\mathcal{N}_{a}(s) \leq \sup _{t}\left|\mathcal{N}_{a}(i t)\right|^{1-s}\left|\mathcal{N}_{a}(1+i t)\right|^{s} \leq C\|v\|_{\left[\mathcal{H}_{0}, \mathcal{H}_{1}\right]_{s}}^{2} .
$$

Thus a constant $C$ exists such that, for any $a$,

$$
\sum_{p=0}^{N} \int_{1}^{a} \lambda^{2 s} d \mu_{p}\left(A_{p} v, A_{p} v\right) \leq C\|v\|_{\left[\mathcal{H}_{0}, \mathcal{H}_{1}\right]_{s}}^{2} .
$$

By definition of $\mathcal{H}_{s, p}$ and using that

$$
\|w\|_{\mathcal{H}_{s, p}}^{2}=\int_{1}^{\infty} \lambda^{2 s} d \mu_{p}(w, w)
$$

we infer by passing to the limit when $a$ tends to infinity in (3.27) that

$$
\sum_{p=0}^{N}\left\|A_{p} v\right\|_{\mathcal{H}_{s, p}}^{2} \leq C\|v\|_{\left[\mathcal{H}_{0}, \mathcal{H}_{1}\right]_{s}}^{2} .
$$

This conclude the proof of Lemma 3.3.

3.4. Conclusion of the proof of Theorem 1.2. Theorem 1.2 follows, observing that the hypothesis of Lemma 3.3 are satisfied with $\mathcal{H}_{0}=L^{2}, \mathcal{H}_{1}=H^{1}(\mathcal{R}), \mathcal{H}_{j, p}$ is the set of $v$ in $\mathcal{H}_{j}$ the support of which is included in $2^{-p} \mathcal{C}$ and $\Lambda_{p}$ is the square root of Dirichlet realization on $2^{-p} \mathcal{C}$ of the operator

$$
\operatorname{Id}+\Delta_{\Sigma} \quad \text { with } \quad \Delta_{\Sigma} \stackrel{\text { def }}{=} \sum_{j, k} \mathcal{R}_{j, k}^{\star} \mathcal{R}_{j, k} .
$$

To be able to apply Lemma 3.3, and then to conclude the proof of Theorem 1.2, it is enough to prove the following proposition. 
Proposition 3.2. A neighbourhood $V$ of $w_{0}$ exists such that the operator $\Delta_{\Sigma}$ is selfadjoint on $L^{2}(V)$ with domain

$$
\left\{v \in L^{2}(V) / \forall\left(j, k, j^{\prime}, k^{\prime}\right) \in\{1, \cdots, 2 d\}^{4}, R_{j, k} v \in L^{2}(V) \quad \text { and } \quad R_{j, k} R_{j^{\prime}, k^{\prime}} v \in L^{2}(V)\right\} .
$$

Proof of Proposition 3.2 Up to an omitted regularization process, it is enough to prove that, for any $v \in \mathcal{D}(V)$,

$$
\sum_{j, k}\left\|R_{j, k} v\right\|_{L^{2}}^{2}+\sum_{j, k, j^{\prime}, k^{\prime}}\left\|R_{j, k} R_{j^{\prime}, k^{\prime}} v\right\|_{L^{2}}^{2} \leq C\left(\|v\|_{L^{2}}^{2}+\left\|\Delta_{\Sigma} v\right\|_{L^{2}}^{2}\right) .
$$

Let us start with the observation that

$$
\begin{aligned}
\sum_{j, k}\left\|R_{j, k} v\right\|_{L^{2}}^{2} \quad a m p ; & \leq a m p ; C \sum_{j, k}\left(R_{j, k} v \mid R_{j, k} v\right)_{L^{2}} \\
a m p ; & \leq a m p ; C \sum_{j, k}\left(R_{j, k}^{\star} R_{j, k} v \mid v\right)_{L^{2}} \\
a m p ; & \leq a m p ; C\left(\Delta_{\Sigma} v \mid v\right)_{L^{2}} \\
a m p ; & \leq a m p ; C\left\|\Delta_{\Sigma} v\right\|_{L^{2}}\|v\|_{L^{2}} .
\end{aligned}
$$

In order to estimate $\left\|R_{j, k} R_{j^{\prime}, k^{\prime}} v\right\|_{L^{2}}$, we are going to proceed as in the proof of Lemma 3.2. Let us write that

$$
R_{j, k} R_{j^{\prime}, k^{\prime}}\left(\varphi_{p} v\right)-\varphi_{p} R_{j, k} R_{j^{\prime}, k^{\prime}} v=\left(R_{j, k} \varphi_{p}\right)\left(R_{j^{\prime}, k^{\prime}} v\right)+\left(R_{j^{\prime}, k^{\prime}} \varphi_{p}\right)\left(R_{j, k} v\right)+\varphi_{p}\left(R_{j, k} R_{j^{\prime}, k^{\prime}} v\right) .
$$

As the coefficients of the vector fields $R_{j, k}$ vanishes on $\Delta_{c}$, we have

$$
\sup _{p, j, k, j^{\prime}, k^{\prime}}\left\|R_{j, k} \varphi_{p}\right\|_{L^{\infty}}+\left\|R_{j, k} R_{j^{\prime}, k^{\prime}} \varphi_{p}\right\|_{L^{\infty}} \leq \infty .
$$

Thus, using (3.29), we have

$$
\left\|\varphi_{p} R_{j, k} R_{j^{\prime}, k^{\prime}} v-R_{j, k} R_{j^{\prime}, k^{\prime}}\left(\varphi_{p} v\right)\right\|_{L^{2}} \leq C c_{p}\left\|\Delta_{\Sigma} v\right\|_{L^{2}}^{\frac{1}{2}}\|v\|_{L^{2}}^{\frac{1}{2}} \quad \text { with } \quad \sum_{p=0}^{\infty} c_{p}^{2}=1 .
$$

We have

$$
R_{j, k} R_{j^{\prime}, k^{\prime}}\left(\varphi_{p} v\right)=R_{j, k, p} R_{j^{\prime}, k^{\prime}, p}\left(\varphi_{0} v\left(2^{p} \cdot\right)\right)
$$

Lemma 4.1 of [4] telles us that the sytems $\left(\mathcal{R}_{j, k, p}\right)_{j, k}$ satisfy the Hörmander condition at order 2 uniformely with respect to $p$ on $\mathcal{C}$. Thus, the classical maximal estimate tells us that

$$
\left\|R_{j, k, p} R_{j^{\prime}, k^{\prime}, p} w\right\|_{L^{2}} \leq C\left(\left\|\sum_{j, k} R_{j, k, p}^{\star} R_{j, k, p} w\right\|_{L^{2}}+\|w\|_{L^{2}}^{2}\right) .
$$

Applied with $w=\varphi_{0} v\left(2^{p} \cdot\right)$, this gives

$$
\begin{aligned}
\left\|R_{j, k} R_{j^{\prime}, k^{\prime}}\left(\varphi_{p} v\right)\right\|_{L^{2}} \quad a m p ; & \leq a m p ; C 2^{p d}\left(\left\|\sum_{j, k} R_{j, k, p}^{\star} R_{j, k, p} \varphi_{0} v\left(2^{p} \cdot\right)\right\|_{L^{2}}+\left\|\varphi_{0} v\left(2^{p} \cdot\right)\right\|_{L^{2}}^{2}\right) \\
a m p ; & \leq a m p ; C\left(\left\|\Delta_{\Sigma}\left(\varphi_{p} v\right)\right\|_{L^{2}}+\left\|\varphi_{p} v\right\|_{L^{2}}\right) .
\end{aligned}
$$

Then (3.31) implies that

$$
\left\|\Delta_{\Sigma}\left(\varphi_{p} v\right)-\varphi_{p} \Delta_{\Sigma} v\right\|_{L^{2}} \leq C c_{p}\left\|\Delta_{\Sigma} v\right\|_{L^{2}}^{\frac{1}{2}}\|v\|_{L^{2}}^{\frac{1}{2}} \quad \text { with } \quad \sum_{p=0}^{\infty} c_{p}^{2}=1 .
$$


Thus, by using (3.30) and (3.32) we infer that

$$
\left\|\varphi_{p} R_{j, k} R_{j^{\prime}, k^{\prime}} v\right\|_{L^{2}} \leq C C c_{p}\left(\left\|\Delta_{\Sigma} v\right\|_{L^{2}}+\|v\|_{L^{2}}\right) \quad \text { with } \quad \sum_{p=0}^{\infty} c_{p}^{2}=1 .
$$

This proves (3.28) and thus Proposition (3.2).

\section{REFERENCES}

[1] H. Bahouri, P. Gérard, and C.-J. Xu, Espaces de Besov et estimations de Strichartz généralisées sur le groupe de Heisenberg, Journal d'Analyse Mathématique, 82, 2000, 93-118.

[2] H. Bahouri et I. Gallagher, Paraproduit sur le groupe de Heisenberg et applications, Revista Mathemàtica Ibero-Americana, 17, 2001, 69-105.

[3] H. Bahouri, J.-Y. Chemin et I. Gallagher, Precised Hardy inequality on $\mathbb{R}^{d}$ and on the Heisenberg group $\mathbb{H}^{d}$, Séminaire EDP de l'X, 2005

[4] H. Bahouri, J.-Y. Chemin et C.-J. Xu, Trace and trace lifting theorems in weighted Sobolev space, Journal de l'Institut de Mathématiques de Jussieu, 4, 2005, pages 1-44.

[5] S. Baouendi, P. Ebenfelt and L. Rothschild, Real Submanifolds in Complex Space and their Mappings, Princeton University Press, 1999.

[6] J. Bergh and J. Löfström, Interpolation spaces. An introduction, Grundlehren der Mathematischen Wissenschaften, 223, Springer-Verlag, 1976.

[7] S. Berhanu and I. Pesenson, The trace problem for vector field satisfying Hörmander's condition, Mathematische Zeitschrift, 231, 1999, pages 103-122.

[8] J.-M. Bony and J.-Y. Chemin, Espaces fonctionnels associés au calcul de Weyl-Hörmander, Bulletin de la Société Mathématique de France, 122, 1994, pages 77-118.

[9] C. E. Cancelier and J.-Y. Chemin, Sous-ellipticité d'opérateurs intégro-différentiels vérifiant le principe du maximum, Annali della Scuola Normale di Pisa, 20, 1994, pages 299-312.

[10] C. E. Cancelier, J.-Y. Chemin and C.-J. Xu, Calcul de Weyl-Hörmander et opérateurs sous-elliptiques, Annales de l'Institut Fourier, 43, 1993, pages 1157-1178.

[11] J.-Y. Chemin and C.-J. Xu, Inclusions de Sobolev en calcul de Weyl-Hörmander et systèmes souselliptiques, Annales de l'École Normale Supérieure, 30, 1997, pages 719-751.

[12] D. Danielli, N. Garofalo, D-N. Nhieu, Trace inequalities for Carnot-Carathédory spaces and applications, Annali della Scuola Normale di Pisa, 27, 1998, pages 195-252.

[13] M. Derridj, Un problème aux limites pour une classe d'opérateurs du second ordre hypoelliptiques, Annales de l'Institut Fourier 21, 1971, pages 99-148.

[14] M. Derridj, Sur l'inégalité de Poincaré, à support compact, pour un ou plusieur champs de vecteurs, Proc. AMS. 129, V.8, 2331-2342.

[15] B. Franchi, S. Gallot and R.I. Wheeden, Sobolev and isoperimetric inequalities for degenerate metrics, Mathematische Annalen, 300, 1994, pages 557-571.

[16] D. Jerison, The Dirichlet problem for the Kohn-Laplacian on the Heisenberg group, Parts I and II, J. Funct. Analysis, 43, 1981, pages 97-142.

[17] D. Jerison, The Poincaré inequality for vector fields satisfying Hörmander's conditions. Duke Mathematical Journal, 53, 1986, pages 503-523.

[18] D. Huet, Décomposition spectrale et opérateurs, Le Mathématicien 16, Presses Universitaires de France, 1976.

[19] A. Nagel, E.M. Stein and S. Wainger, Balls and metrics defined by vector fields I, basic properties, Acta Mathematica, 155, 1985, pages 103-147.

[20] D. M. Nhieu, Extension for Sobolev spaces on the Heisenberg group, C. R. Acad. Sci. Paris, t. 321, Série I, 1995, pages 1559-1564.

[21] I. Pesenson, The trace Problem and Hardy operator for non-isotropic function spaces on the Heisenberg group, Communications in Partial Differential Equations, 19, 1994, pages 655-976.

[22] L. Rothschild and E. Stein, Hypoelliptic differential operators and nilpotent groups, Acta Mathematica, 137, 1977, pages 247-320.

[23] E.M. Stein, Harnomic Analysis, Princeton University Press, 1993. 
Hajer BAhOURI : Département de Mathématiques, Faculté de Sciences de Tunis 1060 Tunis, Tunisie E-MAIL: HAJER.BAHOuri@Fst.Rnu.tn

Jean-Yves CHEMin : Laboratoire J.-L. Lions, UMR 7598, Université Paris 6, 75230 PARis CEdex 05, France E-MAil: Chemin@ANn.Jussieu.Fr

ChaO-Jiang XU : Université de Rouen, UMR-6085, Laboratoire de Mathématiques 76801 Saint-Etienne du Rouvray, France, E-Mail: ChaO-Jiang.Xu@univ-Rouen.fr 